Books, videos, CD-ROMs, DVDs

and any other relevant items

submitted for a review in the

BDJ should be addressed to:

Kate Maynard,

Assistant Editor,

British Dental Journal

Nature Publishing Group,

4-6 Crinan Street

London,

N1 9XW

\section{INFECTION CONTROL AND MANAGEMENT OF HAZARDOUS MATERIALS FOR THE DENTAL TEAM, 4TH EDITION}

\begin{tabular}{|l|l}
\hline INFECTION & $\begin{array}{l}\text { C. H. Miller, C. J. Palenik } \\
\text { UK: Elsevier }\end{array}$ \\
CONTROL & $\begin{array}{l}\text { price } \mathrm{f27.99;} \mathrm{pp} 384 \\
\text { ISBN 9780323056311 }\end{array}$ \\
\hline &
\end{tabular}

As the title suggests this book is a guide to all aspects of infection control, attempting to be a student resource, a management administrative guide and a clinical manual. For students there are learning objectives, teaching aids, review questions and online supporting resources. The clinical aspect is aimed at the entire dental team, encompassing information for nurses, dentists, managers and laboratory technicians. This book therefore contains an eclectic mix of information and there is a confusing lack of differentiation in the expected knowledge, understanding and practical implementation for the different groups. However, to make this information simple to navigate it is split into three distinct sections and 25 chapters with a consistent, systematic approach. Much of the data are clearly presented in tables and the book is well indexed.

Part one, The Microbial World, explains the rationale behind infection control principles. It contextualises biochemistry and microbiology, subjects notoriously difficult for dental students to understand the relevance to their future careers. However, as neither of these subjects are comprehensively covered, this book could be used as supplementary reading rather than a substitute for traditional textbooks.
Part two, Infection Control, combines basic concepts of infection control with specific step-by-step procedural guidelines. As an American textbook the regulations described in detail and used as a basis for the recommended procedures are not of great relevance in British dental practice. Many of the laws and regulations which govern our practice are directly contradicted by the guidelines laid out in this text, including important differences in waste management, immunisation and instrument processing and storage. However, the sections on personal protection and aseptic techniques are directly applicable and could form a sound basis for construction and implementation of best practice local policies.

Part three, Office Safety, covers administrative aspects of infection control with practical guides to developing comprehensive 'hazard communications' and compiling thorough protocols. It contains thought-provoking chapters covering subjects such as greener infection control and medical tourism. However, this section also covers 'infection control overkill' which states that many of the HTM $01-05^{1}$ recommended practices are 'overkill'. These criticisms include routinely cleaning and disinfecting surfaces after removal of surface covers and routinely disinfecting instruments before sterilisation.

Overall this is an interesting and well written text, however, as it is based on American regulations it is of limited value as a reference for British dental teams.

\section{Ola}

1. Decontamination: Health Technical Memorandum 01-05: Decontamination in primary care dental practices. Department of Health, April 2009.

\section{SLEEP MEDICINE FOR DENTISTS: A PRACTICAL OVERVIEW}

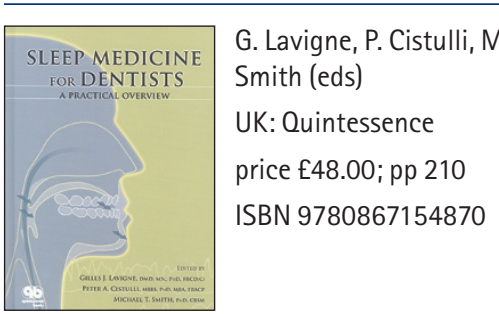

This is a 200 page hardback textbook which gives an overview of sleep medicine from a dental perspective. The editors are experts in the field of sleep medicine and the contributors are a diverse group, involved in either clinical practice or research. The key aim is "to provide a rapid source of practical information to students, practicing dentists and scientists'. It would appear that this is the first comprehensive text of dental sleep medicine, a subject which perhaps does not feature highly in dental education. The editors hope that this text may catalyse the introduction of sleep medicine to dental curricula.

The book is divided into four sections. The first provides an introduction to the subject and encompasses a detailed account of the physiology of sleep and a classification of sleep disorders. Sections II to IV deal with sleep breathing disorders, sleep bruxism and movement disorders and orofacial pain respectively. The ethos of the book is to first understand a disorder and consequently gain an insight into the management strategies which may be employed. As such, the layout of the book is ordered and logical at all times. It is worth noting that the content is evidence-based where possible. If there is a dearth of research in a particular area, the authors ensure this is highlighted. Each chapter is well referenced and will allow the enthusiastic 